\title{
Autophagy-activating strategies to promote innate defense against mycobacteria
}

\author{
Yi Sak Kim ${ }^{1,2}$, Prashanta Silwal $\mathbb{D}^{1,2}$, Soo Yeon Kim³ , Tamotsu Yoshimori ${ }^{4,5}$ and Eun-Kyeong Jo (D) 1,2,
}

\begin{abstract}
Mycobacterium tuberculosis (Mtb) is a major causal pathogen of human tuberculosis (TB), which is a serious health burden worldwide. The demand for the development of an innovative therapeutic strategy to treat TB is high due to drug-resistant forms of TB. Autophagy is a cell-autonomous host defense mechanism by which intracytoplasmic cargos can be delivered and then destroyed in lysosomes. Previous studies have reported that autophagy-activating agents and small molecules may be beneficial in restricting intracellular Mtb infection, even with multidrug-resistant Mtb strains. Recent studies have revealed the essential roles of host nuclear receptors (NRs) in the activation of the host defense through antibacterial autophagy against Mtb infection. In particular, we discuss the function of estrogenrelated receptor (ERR) a and peroxisome proliferator-activated receptor (PPAR) a in autophagy regulation to improve host defenses against Mtb infection. Despite promising findings relating to the antitubercular effects of various agents, our understanding of the molecular mechanism by which autophagy-activating agents suppress intracellular Mtb in vitro and in vivo is lacking. An improved understanding of the antibacterial autophagic mechanisms in the innate host defense will eventually lead to the development of new therapeutic strategies for human TB.
\end{abstract}

\section{Introduction}

There remains a high demand for the development of new drugs against human tuberculosis (TB), which accounts for an estimated 1.3 million deaths globally ${ }^{1}$. TB is mainly caused by Mycobacterium tuberculosis (Mtb), a human pathogen that successfully resides in host macrophages and phagocytic cells ${ }^{2-4}$. Macrophages and phagocytes can trigger numerous innate immune signaling pathways, resulting in the activation of effector molecules to combat intracellular parasites, which can exploit host defense strategies through multiple escape mechanisms, leading to the arrest of phagosomal maturation ${ }^{2,4,5}$. Mtb and the host immune system are involved in complicated crosstalk, which requires further investigation. The development of new vaccines and therapeutics against $\mathrm{TB}$

Correspondence: Eun-Kyeong Jo (hayoungj@cnu.ac.kr)

'Department of Microbiology, Chungnam National University School of Medicine, Daejeon 35015, Korea

${ }^{2}$ Department of Infection Control Convergence Research Center, Chungnam National University School of Medicine, Daejeon 35015, Korea

Full list of author information is available at the end of the article. requires a comprehensive understanding of the molecular mechanisms underlying the host-pathogen interactions during mycobacterial infection ${ }^{6,7}$.

Autophagy is an intracellular process involved in the housekeeping function and maintenance of cellular homeostasis in response to diverse stress conditions ${ }^{8,9}$. It is becoming clear that the autophagy pathway is vital in the host defense against infection by various intracellular pathogens, including Mtb, Salmonella enterica serovar Typhimurium, and Listeria monocytogenes through the enhancement of phagolysosome formation ${ }^{10-15}$. This pathway functions as a cell-autonomous defense system that delivers cytoplasmic cargos and bacterial phagosomes for lysosomal degradation ${ }^{10}$. Accumulating evidence has shown that autophagy contributes to innate and adaptive immune pathways in a variety of settings ${ }^{12,14,16,17}$. However, Mtb has evolved numerous strategies to manipulate host innate immune pathways and evade phagosomal acidification $^{2,18-20}$. Furthermore, recent studies have reported that several autophagy genes do not play a critical role in antimycobacterial defense in murine systems 
in vivo ${ }^{21}$. Nevertheless, numerous drugs/agents are able to induce autophagy activation to promote the restriction and eradication of Mtb in vitro and in vivo ${ }^{22}$. Although there are no specific drugs targeting autophagy, the identification of autophagy-activating small molecules/ agents is a promising and new therapeutic target based on host-directed therapy against $\mathrm{TB}^{22-24}$. In this review, we present a brief overview of autophagy/xenophagy during Mtb infection and highlight the autophagy-activating agents/molecules that promote host defense against Mtb. We subsequently focus on important recent studies concerning the discovery of new functions of NRs that promote host autophagy and antimicrobial responses against Mtb infection.

\section{Overview of autophagy in mycobacterial infection}

Autophagy (herein, "macroautophagy") is a multistep process characterized by (1) the initiation of a doublemembrane vesicle phagophore; (2) closure as an autophagosome; and (3) fusion with a lysosome to form an autolysosome capable of degrading intracytoplasmic cargo (Fig. 1) $)^{25}$. During this process, numerous autophagyrelated genes (ATGs), first identified by Dr. Yoshinori Ohsumi $^{26}$, were shown to play essential roles as part of the cellular machinery underlying autophagy ${ }^{27,28}$. In particular, the core machinery of the autophagy process is essential for autophagosome formation. Two ubiquitinlike protein conjugation systems (ATG12 and ATG8/LC3) play critical roles in the formation and ultimate closure of the double-membrane structures of autophagosomes ${ }^{29}$.

Previous studies have shown that the Th1 cytokine Interferon (IFN) $\gamma$ activates autophagy in macrophages, leading to an increase in antimicrobial host defense against Mtb infection ${ }^{12}$. Numerous additional studies have reported that activation of macroautophagy can promote phagosomal acidification and antimicrobial responses in murine and human macrophages, suggesting that autophagy may represent a promising host-targeting therapeutic strategy against Mtb infection ${ }^{22,24,30}$. Notably, a recent study by Kimmey et al. showed that ATG5, but no other autophagy genes, plays a unique role in host protection during Mtb infection in mouse models ${ }^{21}$. Interestingly, this protective effect was not mediated through autophagy activation but through the amelioration of excessive inflammatory responses caused by polymorphonuclear neutrophils ${ }^{21}$. These observations suggest that the contribution of individual autophagy genes alone is not sufficient to control the growth of intracellular Mtb. Overall, further investigation is warranted to understand whether activation of autophagy by small molecules and/or compounds could enhance the inhibition of intracellular Mtb replication in vivo.

Once regarded as a simple, nonspecific catabolic process, autophagy has proven far more sophisticated than originally thought and is capable of targeting and degrading specific cellular components, including mitochondria, endoplasmic reticulum, lysosomes, and even invading bacteria ${ }^{31,32}$. Xenophagy is a form of selective autophagy in which cells are able to target and selectively capture bacteria, including Mtb or Salmonella Typhimurium, for autophagic degradation ${ }^{14,28}$. Specific processes capable of triggering xenophagy include $\mathrm{Mtb}$ phagosomal permeabilization through the ESX-1 secretion system, which can trigger xenophagy activation through ubiquitin-mediated-dependent pathways ${ }^{13}$. Two examples of these pathways are the ubiquitin ligases Parkin and Smurf1, which are involved in the ubiquitination of cytosolic Mtb, followed by its delivery to autophagic machinery ${ }^{13,33}$. The recognition of cytosolic Mtb DNA by the DNA sensor cGAS is required to target Mtb to the ubiquitin-mediated xenophagy pathway ${ }^{34}$. The cGAS-STING pathway is required for type I IFN production, which can compromise host protective immunity against Mtb infection, though the activation of these processes can vary depending on the particular Mtb $\operatorname{strain}^{35,36}$. Under most circumstances, the elimination of intracellular Mtb by xenophagy is considered beneficial to the host cells; however, the excessive activation of xenophagy by an Mtb eis-deletion mutant induced host cell death and failed to elicit any protective effects in vivo ${ }^{37}$. Taken together, these data suggest that xenophagy activation should be coordinated in conjunction with the appropriate immune responses to promote a more rapid resolution of harmful inflammation, increase cell death and limit the spread of infection.

Another type of noncanonical autophagy pathway is LC3-associated phagocytosis (LAP), which has mainly been studied in fungal infections ${ }^{38,39}$. LAP is an essential link between pattern receptor receptors and phagosomal maturation, helping to enhance the effect of antimicrobial peptides on intracellular pathogens and regulate a variety of physiological functions, including the clearance of apoptotic cells, antigen presentation and type I IFN signaling ${ }^{40,41}$. A recent study found that the Mtb CpsA protein contributes to Mtb escape from the LAP pathway by inhibiting the recruitment of NADPH oxidase 2 (NOX2) to the mycobacterial phagosome ${ }^{42}$. This discovery of the Mtb CpsA protein as a key player in the escape from the LAP pathway has highlighted the need to explore mycobacterial effectors and investigate their ability to modulate canonical and noncanonical autophagic processes during infection ${ }^{42}$. The host autophagy protein Rubicon activates LAP, while inhibiting canonical autophagy $^{43}$. It is necessary to clarify the exact role of Rubicon in autophagy and/or LAP activation during Mtb infection. A schematic overview of autophagy activation during Mtb infection is shown in Fig. 1. In addition, future studies are needed to elucidate the relationship between 


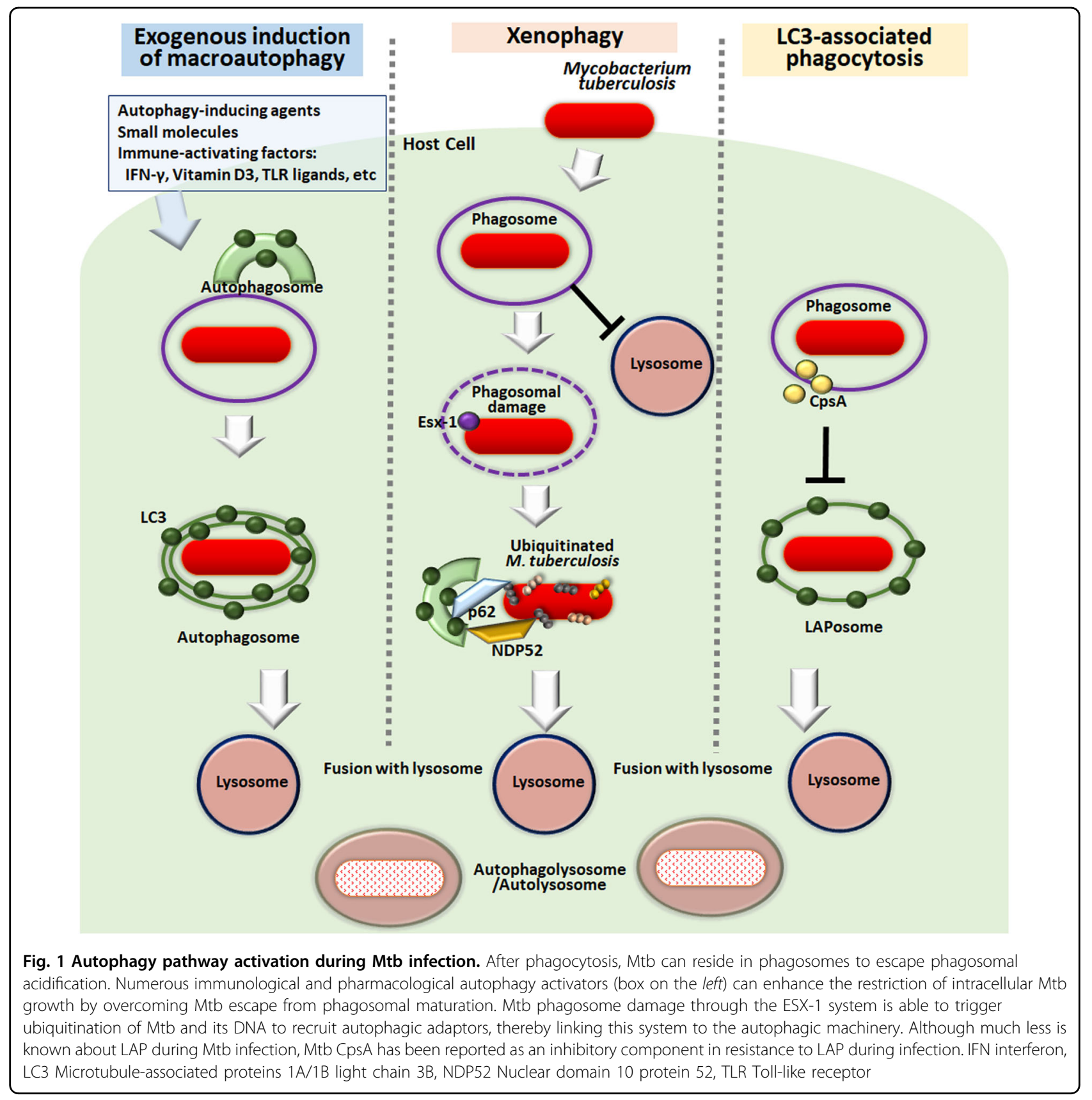

canonical autophagy and LAP in shaping host protective immune responses during Mtb infection.

\section{Promotion of antimycobacterial host defense by autophagy-activating drugs/reagents}

Mtb and many other pathogens employ numerous strategies to inhibit autophagy ${ }^{2,19,44,45}$. Here, we discuss how the treatment of autophagy-activating agents promotes antimicrobial host defenses in vitro and in vivo by overcoming the ability of bacteria to block xenophagy and dampening excessive inflammation during infection (Table 1).

Previous studies have shown that rapamycin, small molecule enhancers of rapamycin (SMER), vitamin D, interferon- $\gamma$, metformin, and 4-phenylbutyrate (PBA) displayed antimicrobial activity against Mtb in human or murine macrophages by enhancing the activation of the autophagy pathway ${ }^{12,46-52}$. In human macrophages, a link between vitamin D-induced autophagy and human cathelicidin microbial peptide (LL-37) has been 


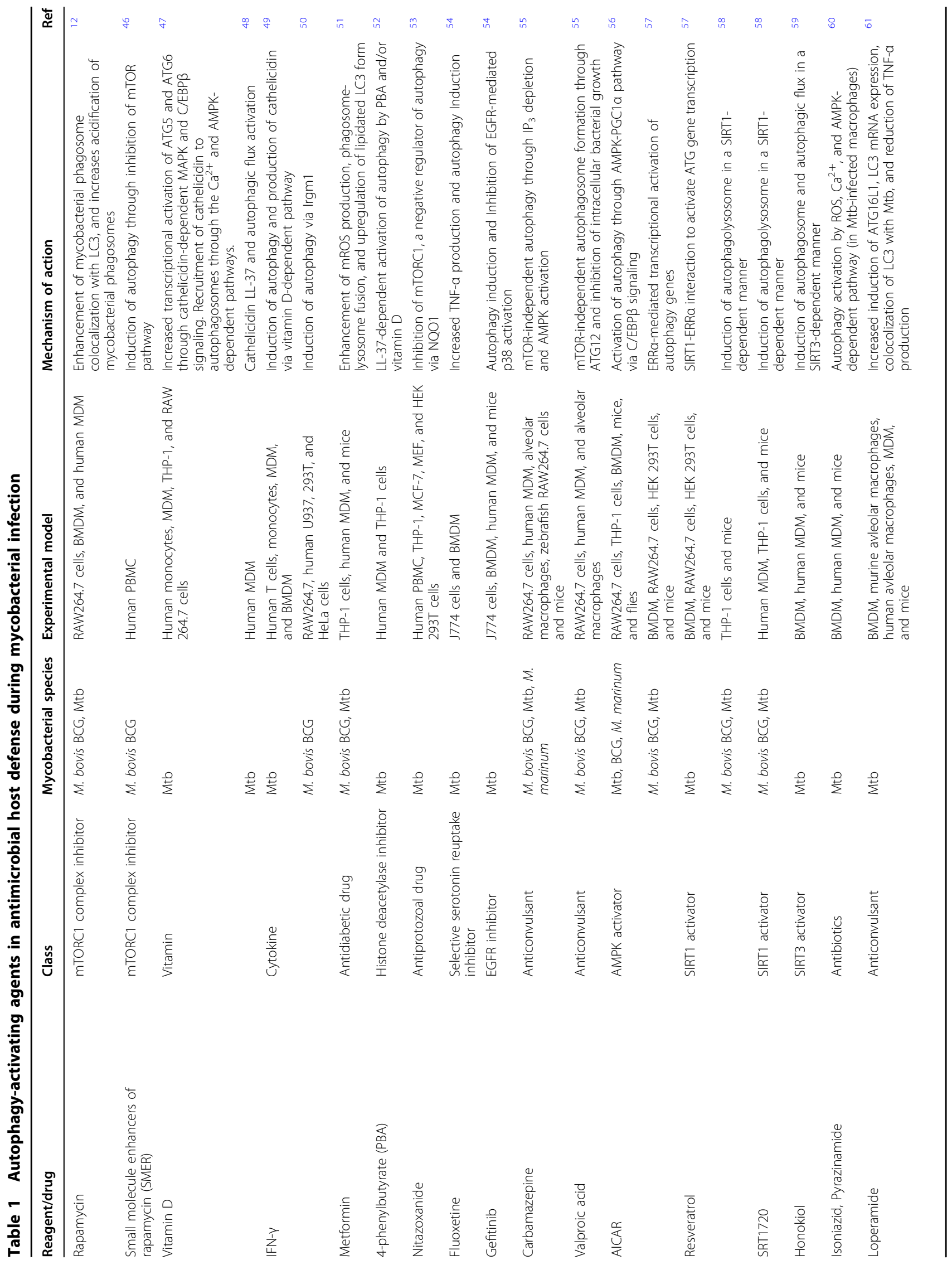




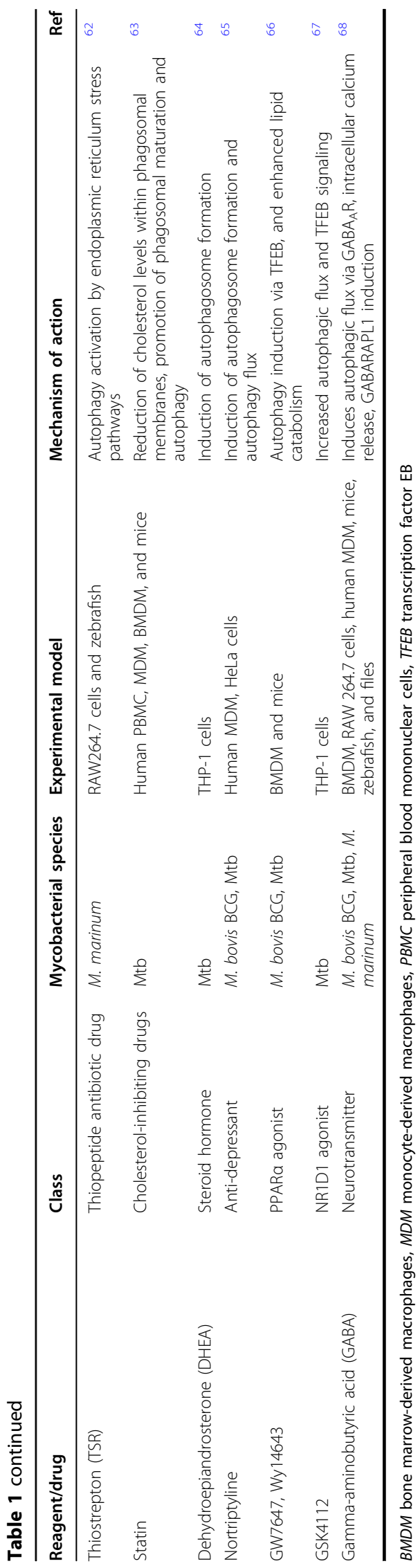

demonstrated $^{47,52}$. Interestingly, PBA and the active form of vitamin D3 $\left(1,25[\mathrm{OH}]_{2} \mathrm{D} 3\right)$ were shown to improve intracellular killing of $\mathrm{Mtb}$ in human macrophages through LL-37 expression and autophagy ${ }^{52}$.

Several pharmacologic agents have been identified for their ability to induce autophagy to promote antimicrobial effects against Mtb infection. For example, the antiprotozoal drug nitazoxanide and its analogs activate autophagosome formation and mTORC1 inhibition, thus restricting Mtb proliferation in vitro ${ }^{53}$. In addition, a chemical screening study using a high-content microscopic assay identified small molecules that inhibit mycobacterial growth in macrophages by targeting host autophagy activation. It was noted that both fluoxetine (a selective serotonin reuptake inhibitor) and gefitinib (an inhibitor of the epidermal growth factor receptor) activate autophagy and reduce Mtb growth in macrophages and in vivo ${ }^{54}$. Another study with cell-based screening of FDA-approved drugs ascertained that the anticonvulsant carbamazepine and valproic acid enhanced mTOR-independent autophagic killing of Mtb in human macrophages ${ }^{55}$. Recent studies have revealed that AMPK activator (5-Aminoimidazole-4-carboxamide 1- $\beta$-Dribofuranoside, AICAR), sirtuin (SIRT) 1 activator (resveratrol, RSV or SRT1720) or a SIRT3 activator (Honokiol) were beneficial for promoting host defenses against mycobacterial infection through autophagy induction, AMPK activation or reduced inflammation ${ }^{56-59}$.

While host-directed therapy has recently emerged as a new therapeutic strategy for the treatment of human TB, accumulating evidence strongly suggests that antimycobacterial antibiotics exert activities through dual modes, acting on both intracellular bacteria and host autophagy activation ${ }^{60}$. The induction of autophagy by treating macrophages with isoniazid and pyrazinamide was required for successful chemotherapeutic effects against intracellular Mtb. The mechanisms of autophagy activation involved the antibiotic-mediated triggering of hydroxyl radicals and cellular reactive oxygen species in Mtb-infected macrophages ${ }^{60}$. Accumulating evidence suggests that drug repurposing, based on autophagy activation, shows promise in the development of new host-directed therapeutics against Mtb infection. Carbamazepine, loperamide, and valproic acid induce ATG expression and autophagy, which are associated with the control of the intracellular growth of Mtb in murine alveolar cells and alveolar macrophages ${ }^{61}$. Recently, thiostrepton (TSR), a thiopeptide antibiotic possessing a quinaldic acid moiety, has been shown to have a dual action on direct targeting to the bacterial ribosome and the induction of ER stress-mediated autophagy to promote the elimination of intracellular mycobacteria ${ }^{62}$. The cholesterol-lowering drugs, statins showed beneficial effects against intracellular Mtb growth through the promotion of phagosomal maturation and autophagy 
activation $^{63}$. In addition, the immunomodulatory drug, dehydroepiandrosterone (DHEA) was beneficial in controlling Mtb load through an autophagy mechanism, which contributes to the clearance of Mtb and the prevention of tissue damage ${ }^{64}$. Moreover, the FDA-approved antidepressant drug, nortriptyline can increase autophagosome formation and xenophagic flux against mycobacteria through the synergistic activation of autophagy with IFN- $\gamma^{65}$. Peroxisome proliferator-activated receptor (PPAR) $\alpha$ agonists (GW7647 and Wy14643) and NR subfamily 1, group D, member 1 (NR1D1) agonist (GSK4112) enhance xenophagic flux via transcription factor EB (TFEB) signaling ${ }^{66,67}$. In our recent report, the major inhibitory neurotransmitter, gamma-aminobutyric acid (GABA) promotes antimicrobial responses and autophagy activation through macrophage type A GABA receptor $\left(G_{A B A_{A}} R\right)$, intracellular calcium release, and the GABA type A receptor-associated protein-like $1^{68}$. Together, these drugs or agents may act as new therapeutics of host-induced autophagy, thereby enhancing host protection against TB.

\section{Nuclear receptors and autophagy in mycobacterial infection}

NRs are important for innate immune responses to control inflammatory responses and infection ${ }^{69}$. In recent reports, emerging evidence suggests that several NRs play critical roles in autophagy activation to promote the innate host defense against mycobacterial infection. The vitamin D-mediated beneficial effects on the restriction of intracellular Mtb growth in macrophages have been studied; however, additional clinical trials of vitamin D-adjunctive therapies for TB are needed to consider all genetic variants ${ }^{23,70,71}$. NR1D1, an orphan NR, also exerts antimycobacterial effects through the reinforcement of autophagic flux and lysosome biogenesis in human macrophages ${ }^{67}$. We recently showed that orphan NR, estrogen-related receptor $\alpha$ (ERR $\alpha$; NR3B1, ERR1, ESRRA), promotes macrophage autophagy in response to various autophagy stimulators, including AICAR and $\mathrm{RSV}^{57}$. In addition, other studies have reported a role for PPAR $\alpha$ in the activation of host defenses in macrophages through autophagy and lysosomal biogenesis ${ }^{66}$. In a recent study of the expression profile of NRs in Mtbinfected macrophages or dendritic cells ${ }^{72}$, several NRs, such as N4a3 and Rora, were identified. Given the findings that numerous NRs appear to be involved in the regulation of autophagy in host cells, future studies are needed to investigate the novel functions of new NRs and their complex interplay with Mtb in the context of autophagy. In this review, we focus on recent studies of the functions of two NRs, ERR $\alpha$ and PPAR $\alpha$.

\section{ERRa and autophagy}

$E R R \alpha$ is the first orphan family member of NRs in which the physiological ligands have not been identified. ERR $\alpha$, along with other members of the ERRs, does not bind estrogens and preferentially binds to an estrogen-related response element (ERRE) to regulate target genes containing these binding elements in their promoter/enhancer regions ${ }^{73}$. Previous functional studies have shown that ERR $\alpha$ plays a transcriptional activating role through an interaction with the transcriptional coactivator PPAR $\gamma$ coactivator-1 $\alpha(\mathrm{PGC}-1 \alpha)^{74,75}$. ERR $\alpha$ function has been widely studied in the regulation of mitochondrial and metabolic gene transcription, particularly in muscle differentiation, thermogenesis, and in heart and bone functions ${ }^{76}$. Previous works have shown that ERR $\alpha$ is a central regulator of innate immune function, including the regulation of toll-like receptor-induced inflammatory responses and antimicrobial responses against intracellular bacterial infection ${ }^{77,78}$. Recently, a new function of $E R R \alpha$ was revealed in the negative regulation of antiviral responses through the inhibition of type-I interferon signaling $^{79}$.

The involvement of ERR $\alpha$, in cooperation with PGC- $1 \alpha$, in the mitochondrial quality control and regulation of autophagy has been shown ${ }^{80}$. ERR $\alpha$ deficiency was associated with incomplete autophagy and necrotic cell death in adrenocortical cancer through the control of bioenergetic metabolism ${ }^{81}$. Thyroid hormone induces ERR $\alpha$, which is essential in the regulation of DRP1-mediated mitochondrial fission and mitophagy through the expression of autophagy-initiating kinase ULK $1^{82}$.

Notably, ERR $\alpha$ was found to be a key transcriptional regulator of numerous ATGs, including ATG5, ATG6, and ATG16L1, which contain ERR response elements in their promoter/enhancer regions ${ }^{57}$. Although ERR $\alpha$ has no physiological ligands, AMPK and SIRT1 activation enhances the induction of ERR $\alpha$ mRNA and proteins, thereby enhancing the formation of autophagosomes and autophagic flux in macrophages. In addition, ERR $\alpha$ plays a posttranslational regulatory role through the deacetylation of several autophagy proteins, including ATG5, ATG6, and ATG7, all of which are regulated through interactions with SIRT1. Furthermore, ERR $\alpha$-deficient mice show defective antimicrobial and excessive inflammatory responses against mycobacterial infection, indicating that ERR $\alpha$ is a possible target of antimicrobial innate defenses during Mtb infection ${ }^{57}$. The transcriptional and posttranslational mechanisms by which ERR $\alpha$ regulates the autophagy pathway are shown in Fig. 2.

\section{PPARa and Autophagy}

The NR PPARs include three isoforms $(\alpha, \delta \text {, and } \gamma)^{83}$, which form heterodimers with retinoid $\mathrm{X}$ receptor and 


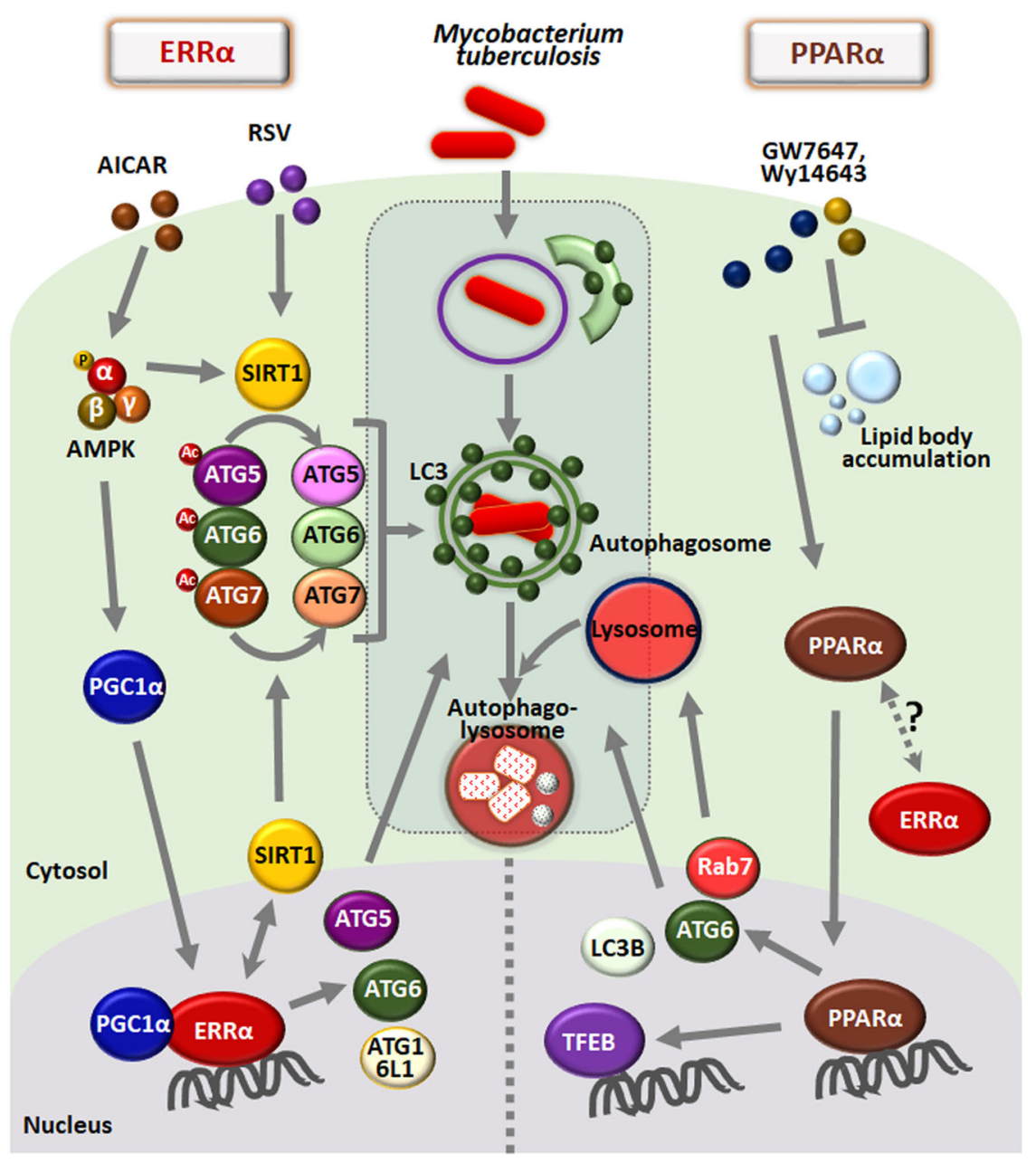

Fig. 2 The roles of ERRa and PPARa in autophagy and host defense against Mtb infection. (Left) ERRa, which is induced by either AMPK or SIRT1 activation, contributes to the induction of autophagosome formation in BMDMs. ERRa is required for the transcriptional activation of several ATGs containing ERR response elements in the promoters. In addition, the cooperation of ERRa with SIRT1 promotes the deacetylation of ATG5, ATG6, and ATG7, thereby activating autophagy at the posttranslational level. ERRa-mediated autophagy activation results in increased phagosomal maturation and antimicrobial responses during Mtb infection. (Right) PPARa, which is activated by PPARa ligands (GW7647 and Wy14643), contributes to enhanced autophagosomal formation and maturation in BMDMs. PPARa is essential for the transcriptional activation of several ATGs, TFEB and lipid catabolism. PPARa reinforces antimicrobial responses to mycobacterial infection by inducing autophagic maturation, TFEB, and lipid catabolism. AICAR, 5-Aminoimidazole-4-carboxamide 1- $\beta$-D-ribofuranoside; RSV resveratrol

bind to AGGTCANAGGTCA, the peroxisome proliferator response element (PPRE), to induce or repress the transcription of target genes ${ }^{84,85}$. The PPAR target genes are mostly involved in metabolic homeostasis in various tissues, including the liver, adipose tissues, heart and muscle ${ }^{85-87}$. Of the three isoforms of PPARs, PPAR $\alpha$ is an important coordinator of lipid metabolism and vascular and inflammatory responses ${ }^{86,87}$. Since PPAR $\alpha$ is critically involved in fatty acid oxidation (FAO), lipid and glucose metabolism, and inflammation, the dysregulation of PPAR $\alpha$ leads to various defects, such as metabolic, cardiovascular and inflammatory diseases ${ }^{88-90}$. In terms of immunological control, PPAR $\alpha$ acts as a critical regulator in immune homeostasis against various inflammatory and infectious stimuli ${ }^{91-95}$. A novel connection between autophagy and PPAR $\alpha$ to influence lipid metabolism and innate immunity has been proposed, where autophagy activation by PPAR $\alpha$ was shown to promote autophagic lipid degradation and innate host defenses ${ }^{66}$. PPAR $\alpha$ activation elevates autophagy, particularly in the transcriptional activation of ATGs ${ }^{66,96}$, which is essential for the regulation of the autophagy process in various tissues and cells ${ }^{97}$. Importantly, there exists a great deal of evidence for crosstalk between PPAR $\alpha$ and TFEB ${ }^{66,98,99}$, which is a master regulator of autophagy, lysosomal function and biogenesis, and lipid 
catabolism $^{98-100}$. Indeed, TFEB is recognized as an important transcriptional factor for the regulation of immune and inflammatory responses ${ }^{100,101}$. Combined with our recent study showing that SIRT3 induces antibacterial autophagy against Mtb infection through PPAR $\alpha^{59}$, the function of PPAR $\alpha$ in the host defense against intracellular Mtb infection might be primarily mediated through its activation of autophagy ${ }^{59,66}$.

Importantly, a recent report showed that PPAR $\alpha$ activation contributes to the enhancement of FAO and lipid catabolism in macrophages during Mtb infection ${ }^{66}$. It would be attractive to examine whether autophagy activation is linked to lipid body inhibition in terms of host defense against Mtb infection. A previous study showed that lipid droplets are delivered to lysosomes via the autophagy pathway, thereby hydrolyzing lipid droplets by the action of lysosomal acid lipase ${ }^{102}$. Thus, autophagy may be required for the regulation of lipid metabolism in macrophages during Mtb infection. PPAR $\alpha$-mediated host defense is summarized in Fig. 2. Gemfibrozil (lipidlowering drug), an FDA-approved PPAR $\alpha$ agonist, has been reported to inhibit the intracellular growth of wildtype and multidrug-resistant $\mathrm{Mtb}$ and suppress the activity of enoyl-CoA reductases ${ }^{103}$. For this reason, gemfibrozil may be a potential anti-TB drug candidate; however, it is unclear whether gemfibrozil-mediated antimicrobial responses depend on autophagy activation. It is an open question whether there is crosstalk between PPAR $\alpha$ and ERR $\alpha$ in terms of antimycobacterial host defense. Defining the unique immunological features of autophagy-activating agents based on NR function may represent a rational path for designing improved therapeutics or protective vaccines against TB.

\section{Concluding remarks}

Autophagy activation by diverse exogenous stimuli has now been recognized for its role in antimicrobial host defense and in regulating immune and inflammatory responses during Mtb infection. However, the mechanisms controlling these antimicrobial responses are not completely understood. Accumulating evidence shows that autophagy-activating agents are crucial for innate host defense and for controlling excessive inflammatory responses against Mtb infection. Future studies are warranted to examine the effects of autophagy-modulating agents, used either alone or together with chemotherapeutic drugs, for their antimicrobial effects against Mtb infection in vivo and in clinical trials. Given the recent reports showing that both ERR $\alpha$ and PPAR $\alpha$ modulate antibacterial autophagy, progress is expected in the development of new therapeutic approaches to treat other infectious diseases beyond tuberculosis. An improved understanding of the molecular mechanisms of autophagy-activating agents will eventually lead to the development of novel therapeutic strategies for human TB.

\section{Acknowledgements}

We are indebted to current and past members of our laboratory for discussions and investigations that contributed to this article. This work was supported by the National Research Foundation of Korea (NRF) Grant funded by the Korean Government (Ministry of Science and ICT) (2017R1A5A2015385) and by the framework of international cooperation program managed by NRF of Korea (Grant Number: 2015K2A2A6002008). This research was supported by Chungnam National University Hospital Research Fund, 2017-2018. The authors have no financial conflicts of interest.

\section{Author details}

'Department of Microbiology, Chungnam National University School of Medicine, Daejeon 35015, Korea. ${ }^{2}$ Department of Infection Control Convergence Research Center, Chungnam National University School of Medicine, Daejeon 35015, Korea. ${ }^{3}$ Drug \& Disease Target Research Team, Division of Bioconvergence Analysis, Korea Basic Science Institute (KBSI), Cheongju 28119, South Korea. ${ }^{4}$ Department of Genetics, Osaka University, Osaka 565-0871, Japan. ${ }^{5}$ Department of Intracellular Membrane Dynamics, Graduate School of Frontier Biosciences, Osaka University, Osaka 565-0871, Japan. ${ }^{6}$ Department of Medical Science, Chungnam National University School of Medicine, Daejeon 35015, Korea

Conflict of interest

The authors declare that they have no conflict of interest.

\section{Publisher's note}

Springer Nature remains neutral with regard to jurisdictional claims in published maps and institutional affiliations.

Received: 18 March 2019 Revised: 3 April 2019 Accepted: 22 May 2019. Published online: 11 December 2019

\section{References}

1. World Health Organization Global tuberculosis report 2018. (World Health Organization, Geneva, 2018).

2. Fratti, R. A., Chua, J., Vergne, I. \& Deretic, V. Mycobacterium tuberculosis glycosylated phosphatidylinositol causes phagosome maturation arrest. Proc. Natl Acad. Sci. USA 100, 5437-5442 (2003).

3. Deretic, V., Via, L. E., Fratti, R. A. \& Deretic, D. Mycobacterial phagosome maturation, rab proteins, and intracellular trafficking. Electrophoresis 18, 2542-2547 (1997).

4. Ehrt, S. \& Schnappinger, D. Mycobacterial survival strategies in the phagosome: defence against host stresses. Cell. Microbiol. 11, 1170-1178 (2009).

5. Via, L. E. et al. Arrest of mycobacterial phagosome maturation is caused by a block in vesicle fusion between stages controlled by rab5 and rab7. J. Biol. Chem. 272, 13326-13331 (1997).

6. Hoffmann, E., Machelart, A., Song, O. R. \& Brodin, P. Proteomics of mycobacterium infection: moving towards a better understanding of pathogendriven immunomodulation. Front. Immunol. 9, 86 (2018).

7. Weiss, G. \& Schaible, U. E. Macrophage defense mechanisms against intracellular bacteria. Immunol. Rev. 264, 182-203 (2015).

8. Nakamura, S. \& Yoshimori, T. New insights into autophagosome-lysosome fusion. J. Cell Sci. 130, 1209-1216 (2017).

9. Yin, Z., Pascual, C. \& Klionsky, D. J. Autophagy: machinery and regulation. Micro. Cell 3, 588-596 (2016).

10. Yoshimori, T. \& Amano, A. Group a Streptococcus: a loser in the battle with autophagy. Curr. Top. Microbiol. Immunol. 335, 217-226 (2009).

11. Yuk, J. M., Yoshimori, T. \& Jo, E. K. Autophagy and bacterial infectious diseases. Exp. Mol. Med. 44, 99-108 (2012).

12. Gutierrez, M. G. et al. Autophagy is a defense mechanism inhibiting BCG and Mycobacterium tuberculosis survival in infected macrophages. Cell $\mathbf{1 1 9}$, 753-766 (2004).

13. Manzanillo, P. S. et al. The ubiquitin ligase parkin mediates resistance to intracellular pathogens. Nature 501, 512-516 (2013). 
14. Gomes, L. C. \& Dikic, I. Autophagy in antimicrobial immunity. Mol. Cell 54, 224-233 (2014).

15. Siqueira, M. D. S., Ribeiro, R. M. \& Travassos, L. H. Autophagy and its interaction with intracellular bacterial pathogens. Front Immunol. 9, 935 (2018).

16. Jang, Y. J., Kim, J. H. \& Byun, S. Modulation of autophagy for controlling immunity. Cells 8, E138 (2019).

17. Delgado, M. A., Elmaoued, R. A., Davis, A. S., Kyei, G. \& Deretic, V. Toll-like receptors control autophagy. EMBO J. 27, 1110-1121 (2008).

18. Liu, C. H., Liu, H. \& Ge, B. Innate immunity in tuberculosis: host defense vs pathogen evasion. Cell. Mol. Immunol. 14, 963-975 (2017).

19. Romagnoli, A. et al. ESX-1 dependent impairment of autophagic flux by Mycobacterium tuberculosis in human dendritic cells. Autophagy $\mathbf{8}$ 1357-1370 (2012).

20. Chandra, P. et al. Mycobacterium tuberculosis Inhibits RAB7 recruitment to selectively modulate autophagy flux in macrophages. Sci. Rep. 5, 16320 (2015)

21. Kimmey, J. M. et al. Unique role for ATG5 in neutrophil-mediated immunopathology during M. tuberculosis infection. Nature 528, 565-569 (2015).

22. Gupta, A., Misra, A. \& Deretic, V. Targeted pulmonary delivery of inducers of host macrophage autophagy as a potential host-directed chemotherapy of tuberculosis. Adv. Drug Deliv. Rev. 102, 10-20 (2016).

23. Paik, S., Kim, J. K., Chung, C. \& Jo, E. K. Autophagy: a new strategy for hostdirected therapy of tuberculosis. Virulence 10, 488-459 (2018).

24. Kaufmann, S. H. E., Dorhoi, A., Hotchkiss, R. S. \& Bartenschlager, R. Hostdirected therapies for bacterial and viral infections. Nat. Rev. Drug Disco. 17, 35-56 (2018).

25. Klionsky, D. J. et al. Guidelines for the use and interpretation of assays for monitoring autophagy (3rd edition). Autophagy 12, 1-222 (2016).

26. Tsukada, M. \& Ohsumi, Y. Isolation and characterization of autophagydefective mutants of Saccharomyces cerevisiae. FEBS Lett. 333, 169-174 (1993).

27. Shibutani, S. T. \& Yoshimori, T. A current perspective of autophagosome biogenesis. Cell Res. 24, 58-68 (2014).

28. Deretic, V. et al. Immunologic manifestations of autophagy. J. Clin. Invest. 125 75-84 (2015).

29. Yang, Z. \& Klionsky, D. J. Mammalian autophagy: core molecular machinery and signaling regulation. Curr. Opin. Cell Biol. 22, 124-131 (2010).

30. Jo, E. K. Autophagy as an innate defense against mycobacteria. Pathog. Dis. 67, 108-118 (2013)

31. Mizumura, K. Choi, A. M. \& Ryter, S. W. Emerging role of selective autophagy in human diseases. Front. Pharm. 5, 244 (2014).

32. Stolz, A., Ernst, A. \& Dikic, I. Cargo recognition and trafficking in selective autophagy. Nat. Cell Biol. 16, 495-501 (2014).

33. Franco, L. H. et al. The ubiquitin ligase Smurf1 FUNCTIONS IN SELECTIVE AUTOPHagy of Mycobacterium tuberculosis and anti-tuberculous host defense. Cell Host Microbe 21, 59-72 (2017).

34. Watson, R. O. et al. The cytosolic sensor cGAS detects Mycobacterium tuberculosis DNA to induce Type I interferons and activate autophagy. Cell Host Microbe 17, 811-819 (2015).

35. McNab, F. W. et al. Type I IFN induces IL-10 production in an IL-27independent manner and blocks responsiveness to IFN-gamma for production of IL-12 and bacterial killing in Mycobacterium tuberculosis-infected macrophages. J. Immunol. 193, 3600-3612 (2014).

36. Wiens, K. E. \& Ernst, J. D. The mechanism for Type I interferon induction by Mycobacterium tuberculosis is bacterial strain-dependent. PLoS Pathog. 12 e1005809 (2016).

37. Shin, D. M. et al. Mycobacterium tuberculosis eis regulates autophagy, inflammation, and cell death through redox-dependent signaling. PLOS Pathog. 6, e1001230 (2010).

38. Sprenkeler, E. G., Gresnigt, M. S. \& van de Veerdonk, F. L. LC3-associated phagocytosis: a crucial mechanism for antifungal host defence against Aspergillus fumigatus. Cell. Microbiol. 18, 1208-1216 (2016).

39. Martinez, J. et al. Molecular characterization of LC3-associated phagocytosis reveals distinct roles for Rubicon, NOX2 and autophagy proteins. Nat. Cell Biol. 17, 893-906 (2015).

40. Henault, J. et al. Noncanonical autophagy is required for type I interferon secretion in response to DNA-immune complexes. Immunity 37, 986-997 (2012).

41. Green, D. R., Oguin, T. H. \& Martinez, J. The clearance of dying cells: table for two. Cell Death Differ. 23, 915-926 (2016).
42. Koster, S. et al. Mycobacterium tuberculosis is protected from NADPH oxidase and LC3-associated phagocytosis by the LCP protein CpsA. Proc. Natl Acad. Sci. USA 114, E8711-E8720 (2017).

43. Wong, S. W., Sil, P. \& Martinez, J. Rubicon: LC3-associated phagocytosis and beyond. FEBS J. 285, 1379-1388 (2018).

44. Sorbara, M. T. \& Girardin, S. E. Emerging themes in bacterial autophagy. Curr. Opin. Microbiol. 23, 163-170 (2015).

45. Yoshikawa, Y. et al. Listeria monocytogenes ActA-mediated escape from autophagic recognition. Nat. Cell Biol. 11, 1233-1240 (2009).

46. Floto, R. A. et al. Small molecule enhancers of rapamycin-induced TOR inhibition promote autophagy, reduce toxicity in Huntington's disease models and enhance killing of mycobacteria by macrophages. Autophagy $\mathbf{3}$ 620-622 (2007).

47. Yuk, J. M. et al. Vitamin D3 induces autophagy in human monocytes/macrophages via cathelicidin. Cell Host Microbe 6, 231-243 (2009).

48. Campbell, G. R. \& Spector, S. A. Vitamin D inhibits human immunodeficiency virus type 1 and Mycobacterium tuberculosis infection in macrophages through the induction of autophagy. PLoS Pathog. 8, el002689 (2012).

49. Fabri, M. et al. Vitamin $D$ is required for IFN-gamma-mediated antimicrobial activity of human macrophages. Sci. Transl. Med. 3, 104 ra102 (2011).

50. Singh, S. B., Davis, A. S., Taylor, G. A. \& Deretic, V. Human IRGM induces autophagy to eliminate intracellular mycobacteria. Science 313, 1438-1441 (2006).

51. Singhal, A. et al. Metformin as adjunct antituberculosis therapy. Sci. Transl. Med. 6, $263 r a 159$ (2014).

52. Rekha, R. S. et al. Phenylbutyrate induces LL-37-dependent autophagy and intracellular killing of Mycobacterium tuberculosis in human macrophages. Autophagy 11, 1688-1699 (2015).

53. Lam, K. K. et al. Nitazoxanide stimulates autophagy and inhibits mTORC1 signaling and intracellular proliferation of Mycobacterium tuberculosis. PLoS Pathog. 8, e1002691 (2012).

54. Stanley, S. A. et al. Identification of host-targeted small molecules that restrict intracellular Mycobacterium tuberculosis growth. PLoS Pathog. 10, e1003946 (2014).

55. Schiebler, M. et al. Functional drug screening reveals anticonvulsants as enhancers of mTOR-independent autophagic killing of Mycobacterium tuberculosis through inositol depletion. EMBO Mol. Med. 7, 127-139 (2015).

56. Yang, C. S. et al. The AMPK-PPARGC1A pathway is required for antimicrobial host defense through activation of autophagy. Autophagy 10, 785-802 (2014).

57. Kim, S. Y. et al. ESRRA (estrogen-related receptor alpha) is a key coordinator of transcriptional and post-translational activation of autophagy to promote innate host defense. Autophagy 14, 152-168 (2018).

58. Cheng, C. Y. et al. Host sirtuin 1 regulates mycobacterial immunopathogenesis and represents a therapeutic target against tuberculosis. Sci. Immunol. 2, eaaj1789 (2017).

59. Kim, T. S. et al. SIRT3 promotes antimycobacterial defenses by coordinating mitochondrial and autophagic functions. Autophagy 15, 1356-1375 (2019).

60. Kim, J. J. et al. Host cell autophagy activated by antibiotics is required for their effective antimycobacterial drug action. Cell Host Microbe 11, 457-468 (2012).

61. Juarez, E. et al. Loperamide restricts intracellular growth of Mycobacterium tuberculosis in lung macrophages. Am. J. Respir. Cell Mol. Biol. 55, 837-847 (2016).

62. Zheng, Q. et al. Thiopeptide antibiotics exhibit a dual mode of action against intracellular pathogens by affecting both host and microbe. Chem. Biol. 22, 1002-1007 (2015).

63. Parihar, S. P. et al. Statin therapy reduces the Mycobacterium tuberculosis burden in human macrophages and in mice by enhancing autophagy and phagosome maturation. J. Infect. Dis. 209, 754-763 (2014).

64. Bongiovanni, B. et al. Effect of cortisol and/or DHEA on THP1-derived macrophages infected with Mycobacterium tuberculosis. Tuberculosis 95, 562-569 (2015).

65. Sundaramurthy, V. et al. Integration of chemical and RNAi multiparametric profiles identifies triggers of intracellular mycobacterial killing. Cell Host Microbe 13, 129-142 (2013).

66. Kim, Y. S. et al. PPAR-alpha activation mediates innate host defense through induction of TFEB and lipid catabolism. J. Immunol. 198, 3283-3295 (2017).

67. Chandra, V., Bhagyaraj, E., Nanduri, R., Ahuja, N. \& Gupta, P. NR1D1 ameliorates Mycobacterium tuberculosis clearance through regulation of autophagy. Autophagy 11, 1987-1997 (2015). 
68. Kim, J. K. et al. GABAergic signaling linked to autophagy enhances host protection against intracellular bacterial infections. Nat. Commun. 9, 4184 (2018).

69. Jin, H. S., Kim, T. S. \& Jo, E. K. Emerging roles of orphan nuclear receptors in regulation of innate immunity. Arch. Pharm. Res 39, 1491-1502 (2016).

70. Bekele, A. et al. Daily adjunctive therapy with vitamin D3 and phenylbutyrate supports clinical recovery from pulmonary tuberculosis: a randomized controlled trial in Ethiopia. J. Intern. Med. 284, 292-306 (2018).

71. Ganmaa, D. et al. High-Dose Vitamin D3 during Tuberculosis Treatment in Mongolia. A Randomized Controlled Trial. Am. J. Respir. Crit. Care Med. 196 628-637 (2017).

72. Saini, A. et al. An accord of nuclear receptor expression in M. tuberculosis infected macrophages and dendritic cells. Sci. Rep. 8, 2296 (2018).

73. Audet-Walsh, E. \& Giguere, V. The multiple universes of estrogen-related receptor alpha and gamma in metabolic control and related diseases. Acta Pharmacol. Sin. 36, 51-61 (2015).

74. Huss, J. M., Kopp, R. P. \& Kelly, D. P. Peroxisome proliferator-activated receptor coactivator-1alpha (PGC-1alpha) coactivates the cardiac-enriched nuclear receptors estrogen-related receptor-alpha and -gamma. Identification of novel leucine-rich interaction motif within PGC-1alpha. J. Biol. Chem. 277 40265-40274 (2002).

75. Giguere, V. Transcriptional control of energy homeostasis by the estrogenrelated receptors. Endocr. Rev. 29, 677-696 (2008).

76. Huss, J. M., Garbacz, W. G. \& Xie, W. Constitutive activities of estrogen-related receptors: Transcriptional regulation of metabolism by the ERR pathways in health and disease. Biochim. Biophys. Acta 1912-1927, 2015 (1852).

77. Yuk, J. M. et al. Orphan nuclear receptor ERRalpha controls macrophage metabolic signaling and A20 expression to negatively regulate TLR-induced inflammation. Immunity 43, 80-91 (2015).

78. Sonoda, J. et al. Nuclear receptor ERR alpha and coactivator PGC-1 beta are effectors of IFN-gamma-induced host defense. Genes Dev. 21, 1909-1920 (2007).

79. He, X. et al. ERRalpha negatively regulates type I interferon induction by inhibiting TBK1-IRF3 interaction. PLoS Pathog. 13, e1006347 (2017).

80. Qi, Z. \& Ding, S. Transcriptional regulation by nuclear corepressors and PGC1alpha: implications for mitochondrial quality control and insulin sensitivity. PPAR Res. 2012, 348245 (2012).

81. Casaburi, I. et al. Estrogen related receptor alpha (ERRalpha) a promising target for the therapy of adrenocortical carcinoma (ACC). Oncotarget $\mathbf{6}$, 25135-25148 (2015).

82. Singh, B. K. et al. Thyroid hormone receptor and ERRalpha coordinately regulate mitochondrial fission, mitophagy, biogenesis, and function. Sci. Signal 11, eaam5855 (2018)

83. Dreyer, $\mathrm{C}$. et al. Control of the peroxisomal beta-oxidation pathway by a novel family of nuclear hormone receptors. Cell 68, 879-887 (1992).

84. Berger, J. \& Moller, D. E. The mechanisms of action of PPARs. Annu. Rev. Med. 53, 409-435 (2002)

85. Michalik, L. et al. International Union of Pharmacology. LXI. Peroxisome proliferator-activated receptors. Pharmacol. Rev. 58, 726-741 (2006).
86. Pawlak, M., Lefebvre, P. \& Staels, B. Molecular mechanism of PPARalpha action and its impact on lipid metabolism, inflammation and fibrosis in nonalcoholic fatty liver disease. J. Hepatol. 62, 720-733 (2015).

87. Lee, W. S. \& Kim, J. Peroxisome proliferator-activated receptors and the heart: lessons from the past and future directions. PPAR Res. 2015, 271983 (2015).

88. Djouadi, F. et al. A gender-related defect in lipid metabolism and glucose homeostasis in peroxisome proliferator- activated receptor alpha- deficient mice. J. Clin. Invest. 102, 1083-1091 (1998).

89. Watanabe, K. et al. Constitutive regulation of cardiac fatty acid metabolism through peroxisome proliferator-activated receptor alpha associated with age-dependent cardiac toxicity. J. Biol. Chem. 275, 22293-22299 (2000).

90. Devchand, P. R. et al. The PPARalpha-leukotriene B4 pathway to inflammation control. Nature 384, 39-43 (1996).

91. Mansouri, R. M., Bauge, E., Staels, B. \& Gervois, P. Systemic and distal repercussions of liver-specific peroxisome proliferator-activated receptor-alpha control of the acute-phase response. Endocrinology 149, 3215-3223 (2008).

92. Huang, W., Eum, S. Y., Andras, I. E., Hennig, B. \& Toborek, M. PPARalpha and PPARgamma attenuate HIV-induced dysregulation of tight junction proteins by modulations of matrix metalloproteinase and proteasome activities. FASEB J. 23, 1596-1606 (2009).

93. Standage, S. W., Caldwell, C. C., Zingarelli, B. \& Wong, H. R. Reduced peroxisome proliferator-activated receptor alpha expression is associated with decreased survival and increased tissue bacterial load in sepsis. Shock 37, 164-169 (2012).

94. Drosatos, K. et al. Inhibition of c-Jun-N-terminal kinase increases cardiac peroxisome proliferator-activated receptor alpha expression and fatty acid oxidation and prevents lipopolysaccharide-induced heart dysfunction. J. Biol. Chem. 286, 36331-36339 (2011).

95. Penas, F. et al. Treatment in vitro with PPARalpha and PPARgamma ligands drives M1-to-M2 polarization of macrophages from T. cruzi-infected mice. Biochim Biophys. Acta 1852, 893-904 (2015).

96. Lee, J. M. et al. Nutrient-sensing nuclear receptors coordinate autophagy. Nature 516, 112-115 (2014).

97. Kuma, A., Komatsu, M. \& Mizushima, N. Autophagy-monitoring and autophagy-deficient mice. Autophagy 13, 1619-1628 (2017).

98. Settembre, C. et al. TFEB controls cellular lipid metabolism through a starvation-induced autoregulatory loop. Nat. Cell Biol. 15, 647-658 (2013).

99. Ghosh, A. et al. Activation of peroxisome proliferator-activated receptor alpha induces lysosomal biogenesis in brain cells: implications for lysosomal storage disorders. J. Biol. Chem. 290, 10309-10324 (2015).

100. Brady, O. A., Martina, J. A. \& Puertollano, R. Emerging roles for TFEB in the immune response and inflammation. Autophagy 14, 181-189 (2018).

101. Visvikis, O. et al. Innate host defense requires TFEB-mediated transcription of cytoprotective and antimicrobial genes. Immunity 40, 896-909 (2014).

102. Ouimet, M. et al. Autophagy regulates cholesterol efflux from macrophage foam cells via lysosomal acid lipase. Cell Metab. 13, 655-667 (2011).

103. Reich-Slotky, R. et al. Gemfibrozil inhibits Legionella pneumophila and Mycobacterium tuberculosis enoyl coenzyme A reductases and blocks intracellular growth of these bacteria in macrophages. J. Bacteriol. 191, 5262-5271 (2009). 